\title{
Trends in Body Mass of Ducks over Time: The Hypotheses in Guillemain et al. Revisited
}

\author{
Gunnar Gunnarsson, Johan Elmberg, \\ Jonas Waldenström
}

Received: 6 December 2010/Accepted: 14 December 2010/Published online: 20 January 2011

This comment was not peer reviewed.

In a recent issue of AMBIO, Guillemain et al. (2010) (hereafter GUN) showed that wintering Mallard (Anas platyrhynchos) and Teal (Anas crecca) in France increased in body mass from 1952-1969 to 2002-2008, but that body size did not change. These results were used to address four hypotheses: that the increase in body mass was due to (1) more benign winter climate in France, (2) a shift in the distribution of subpopulations, (3) habitat management and (4) supplemental stocking of hand-reared ducks. The authors argued that their data support the climate change and local habitat management hypotheses, but not the others. This was because (1) of a lack of studies supporting a shift in winter distributions and (2) that both Mallard and Teal showed similar trends, whereas effects of restocking programs should only affect Mallard. We agree with these interpretations per se and think the hypotheses are of great current concern. In the present contribution, we address the same hypotheses with similar data, but from a different flyway to test the generality of the patterns. The results presented here are from ducks belonging to 'the Northwest European flyway', as compared to 'the Mediterranean flyway' in the original contribution. A mark-recovery analysis of the same ducks as in this study show very few recoveries from the Mediterranean flyway, thus confirming a clear separation between populations (Gunnarsson et al. unpublished results).

The present analysis is based on body mass data from Mallard and Teal ringed at Ottenby, SE Sweden $\left(56^{\circ} 13^{\prime} \mathrm{N}\right.$

The online version of the article commented upon can be found at doi: 10.1007/s13280-010-0020-9 $16^{\circ} 27^{\prime} \mathrm{E}$; see Latorre-Margalef et al. 2009 for trapping details), an important stopover site for waterfowl migrating over the southern Baltic Sea. We contrasted two time periods: 1967-1974 (hereafter early period) and 2002-2008 (recent period). The latter is the same period as in GUN, whereas the early period is somewhat later. We set out to analyze data in the same way as in GUN. However, our analysis was restricted to body mass since data on body size are not available from the early period. Moreover, withinseason effects could not be analyzed for Teal because most individuals leave Ottenby by the end of September. No ducks are normally caught at Ottenby in January-March (i.e. the late winter period in GUN), and we thus analyzed Mallard data from August-September (not analyzed in GUN), October-November and December (similar to GUN). Variances differed between groups (i.e. sex: males and females; age: juvenile [first calendar year] and adult [second calendar year or older]) and we therefore used Mann-Whitney $U$ tests to contrast early and recent samples.

Data from 6,041 Mallard and 607 Teal were analyzed (see Fig. 1 for group-specific sample sizes). In Mallard males, body mass was higher in the recent period in all comparisons except adults in December (which was also the smallest sample) (Fig. 1). Likewise, juvenile females caught in August-November were heavier in recent years, but not those caught in December (Fig. 1). Adult Mallard females did not show any body mass change. Interestingly, Teal body mass did not differ between the two periods in any group (Fig. 1). Contrasting our study and GUN, patterns are thus generally the same for Mallard but different for Teal.

GUN more or less rejected the hypotheses that increased body mass in wintering French ducks was due to stocking programs or to a shift in the distribution of subpopulations. They instead found support for the hypotheses that climate 
Fig. 1 Mean body mass (with $95 \%$ confidence intervals) for different sex and age groups of Mallard and Teal. Data from 1967 to 1974 (early period) are shown as closed circles and from 2002 to 2008 (recent period) as open circles. Three within-season periods (AugustSeptember, October-November, December) are considered for Mallard, whereas only AugustSeptember data are analyzed for Teal. Significant differences are marked with either $* * P<0.01$ or $* * * P<0.001$. Sample sizes for Mallard in the three subsequent within-season periods and in early versus recent periods are 102, 194, 55 versus 266, 314, 39 for adult males, 213, 572, 207 versus 484, 1058, 160 for juvenile males, 26, 72, 19 versus 36, 277 , 45 for adult females and 168 , 427, 128 versus 141, 874, 164 for juvenile females.

Corresponding data for Teal are 32 versus 20 for adult males, 204 versus 19 for juvenile males, 34 versus 9 for adult females and 268 versus 21 for juvenile females
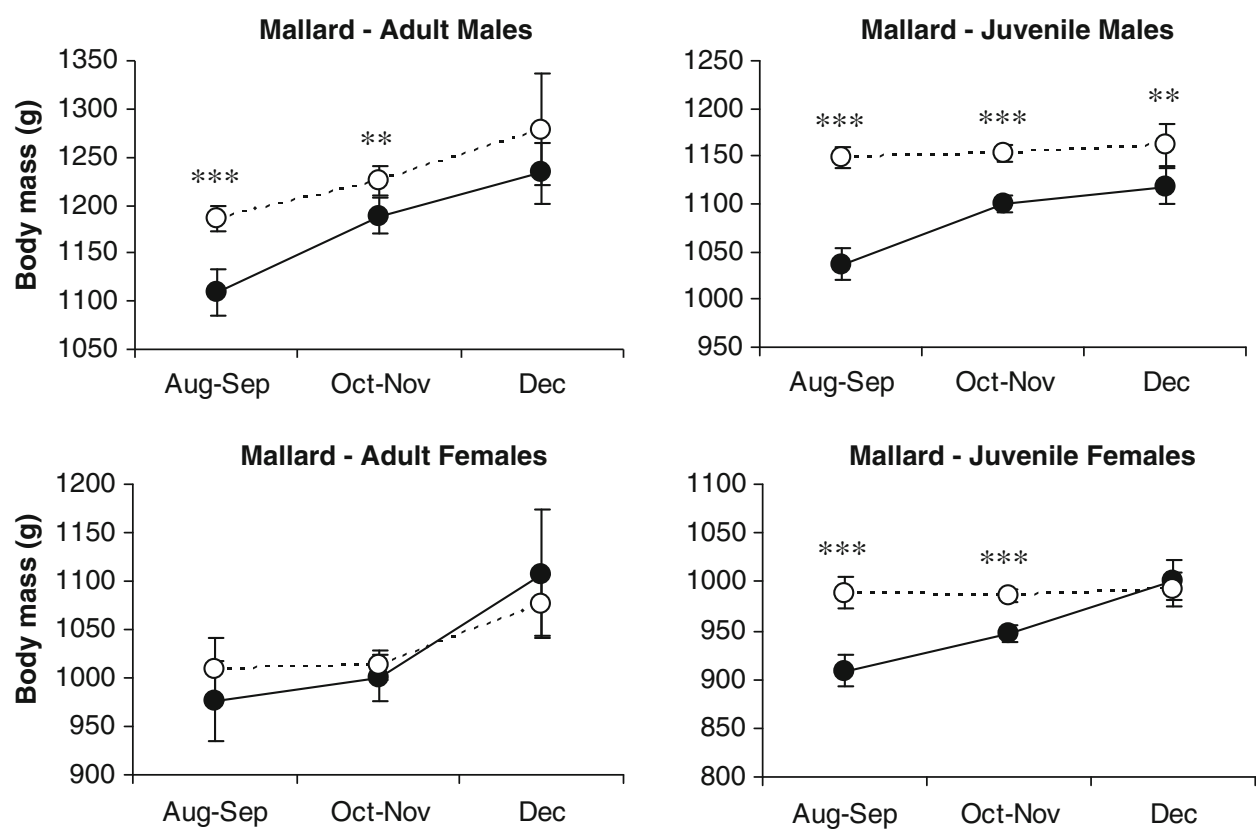

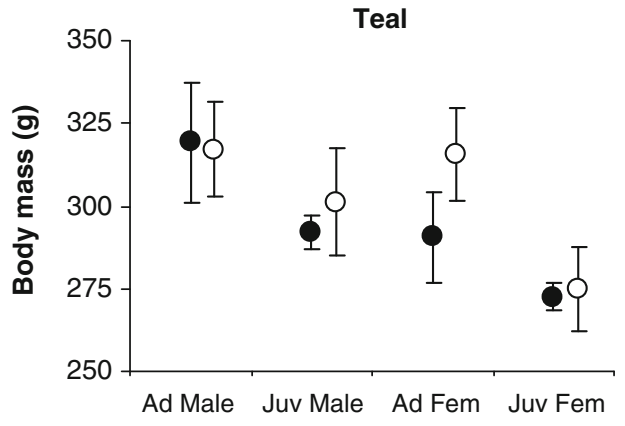

warming and/or habitat management caused the change. We argue that the picture may be different in the adjacent flyway. Data presented here rather support the hypothesis that stocking programs may have made wild Mallards heavier, assuming that hand-reared Mallards are bigger than wild (cf. GUN). This is because we observed increased body mass in most Mallard groups (the lack of difference in adult females may be due to smaller sample sizes in these groups), but not at all in Teal, a species not subjected to stocking programs.

Although not mutually exclusive from the stocking effect hypothesis, neither can a shift in the distribution of subpopulations be rejected as a cause of enhanced body mass in Mallard. The Mallard in the Northwest European flyway has indeed shortened its migration distance in winter compared to earlier decades (Sauter et al. 2010; Gunnarsson et al. unpublished results). It is thus possible that shorter migration distance leads to higher body mass, because resources can be allocated to body mass improvement instead of migration expenses.
In summary, we would like to bring the rejected hypotheses in GUN back to the table. We argue that increased body mass in Mallard may in part result from introgression by farmed-released birds and/or a shift in wintering areas in the Northwest European flyway. In contrast to the Mallard, there is no evidence that Teal has shifted its winter distribution, and the species is surely not affected by stocking. This could explain why body mass has increased in Mallard but not in Teal. We also argue that it is important to address multiple flyways when studying extrinsic effects on biometrics in migratory birds, and look forward to other studies addressing these questions elsewhere.

\section{REFERENCES}

Guillemain, M., J. Elmberg, M. Gauthier-Clerc, G. Massez, R. Hearn, J. Champagnon, and G. Simon. 2010. Wintering French Mallard and Teal are heavier and in better body condition than 30 years ago: Effects of a changing environment? AMBIO 39: 170-180. 
Latorre-Margalef, N., G. Gunnarsson, V.J. Munster, R.A.M. Fouchier, A.D.M.E. Osterhaus, J. Elmberg, B. Olsen, A. Wallensten, et al. 2009. Effects of influenza A virus infection on migrating mallard ducks. Proceedings of the Royal Society B 276: 1029-1036.

Sauter, A., F. Korner-Nievergelt, and L. Jenni. 2010. Evidence of climate change effects on within-winter movements of European Mallards Anas platyrhynchos. Ibis 152: 600-609.

Gunnar Gunnarsson $(\bowtie)$

Address: Aquatic Biology and Chemistry, Kristianstad University,

Kristianstad, Sweden.

e-mail: gunnar.gunnarsson@hkr.se
Johan Elmberg

Address: Aquatic Biology and Chemistry, Kristianstad University, Kristianstad, Sweden.

\section{Jonas Waldenström}

Address: Section for Zoonotic Ecology and Epidemiology, School of Natural Sciences, Linnaeus University, Kalmar, Sweden. 